\title{
Editorial: The Vasopressin System and Behavior
}

\author{
Heather K. Caldwell ${ }^{1 *}$ and Aras Petrulis ${ }^{2}$ \\ ${ }^{1}$ Laboratory of Neuroendocrinology and Behavior, Department of Biological Sciences and School of Biomedical Sciences, \\ Kent State University, Kent, OH, United States, ${ }^{2}$ Neuroscience Institute, Georgia State University, Atlanta, GA, United States
}

Keywords: vasopressin, oxytocin, vasotocin, behavior, brain

\section{The Editorial on the Research Topic}

\section{The Vasopressin System and Behavior}

The awarding of the 1955 Nobel Prize in Chemistry to Vincent du Vigneaud, in part for his isolation and synthesis of the neuropeptide arginine vasopressin (Avp), ushered in a new era of research focused on the ways in which Avp (and its evolutionary precursor vasotocin (Avt)) regulate physiology and behavior $(1,2)$. While early studies focused primarily on the peripheral effects of these neuropeptides, it soon emerged that Avp, and its homologs, are important neuromodulators of behavior; a PubMed search indicates a steady rise in the number of published papers on this topic each year since the mid-1950s, with over 120 papers published per year since 2012. No doubt this continued enthusiasm is due, in part, to the evolutionarily ancient and highlyconserved nature of Avp-like molecules across vertebrate taxa (3). As a result, there is now a tremendous richness in this literature, both in species breadth and mechanistic depth. It was with this in mind that we organized this research topic on "The Vasopressin System and Behavior," in which we hoped to bring together a diversity of perspectives outlining areas of consensus and divergence, as well as to facilitate discussion.

Within this collection, numerous reviews and empirical papers explore the complexity of the Avp and Avt systems in the context of different behavioral systems and taxa. From these papers, several broad themes and some "calls to action" have emerged. First, although it is clear that Avp/Avt are key regulators of social and emotional behavior [e.g., (4-7)], there is a lack of consensus regarding the contexts under which they modulate behavior. Thus, this remains an area requiring even deeper scrutiny. For example, Carter reviews how complex interactions between the Avp and oxytocin systems can affect an animal's responses within differing emotional and social contexts. Second, a broader look at how Avp/Avt influences brain systems, rather than just individual brain regions, is required for further progress. This is underscored by papers from Ophir and Phelps et al. that outline the potential for Avp and oxytocin to alter cortical and hippocampal dynamics underlying complex social space use. Third, even though sex-differences in response to Avp have been frequently noted in the literature [e.g., (8-11)], there are still behavioral domains and taxa in which more critical evaluation of sex differences in the Avp/Avt systems are needed Taylor et al.; Wilczynski et al.; Tickerhoof and Smith; Terranova et al. Fourth, as Simmons et al., Murgatroyd et al., and Baran demonstrate, there is an important role for Avp/Avt throughout behavioral development that is currently under-examined. Fifth, as is explored in Caldwell et al. and Terranova et al., continued consideration of receptor dynamics and subtypes that mediate Avp effects will be needed to more fully understand the relationship between Avp release and its locus of action. Sixth, given the ancient origin of Avp/Avt, a continued commitment to explore their roles across species will aid in finding areas of convergence and divergence in behavioral function. This diversity of Avp/Avt behavioral action across different species is nicely illustrated 
by contributions from Baran, Rodriguez-Santiago et al., Wilczynski et al., and Latzman et al. Lastly, the Avp system is clearly important in regulating human and non-human primate behavior, as described by Latzman et al., Murgatroyd et al., Patisaul, Rilling et al., Price et al., Taylor et al. However, compared to the avalanche of data on oxytocin effects in humans, much less is known and understood regarding the how, the where, and the when of Avp effects on primate social behavior. Thus, there is a need for further detailed examinations of Avp's influence on behavior and cognition, especially within the context of human health.

We are hopeful that these diverse and thoughtful papers will be utilized by both new and more seasoned

\section{REFERENCES}

1. du Vigneaud V, Gish DT, Katsoyannis PG. A synthetic preparation possessing biological properties associated with arginine-vasopressin. J Amer Chem Soc. (1954) 76: 4751-2.

2. du Vigneaud V, Lawler HC, Popenoe EA. Enzymic cleavage of glycinamide from vasopressin and a proposed structure for this pressorantidiuretic hormone of the posterior pituitary. J Amer Chem Soc. (1953) 75:4880-1.

3. Caldwell HK, Lee HJ, Macbeth AH, Young WS, 3rd. Vasopressin: behavioral roles of an "original" neuropeptide. Prog Neurobiol. (2008) 84:1-24. doi: 10.1016/j.pneurobio.2007.10.007

4. Goodson JL, Thompson RR. Nonapeptide mechanisms of social cognition, behavior and species-specific social systems. Curr Opin Neurobiol. (2010) 20:784-94. doi: 10.1016/j.conb.2010.08.020

5. Caldwell HK, Albers HE. Oxytocin, vasopressin, and the motivational forces that drive social behaviors. Curr Top Behav Neurosci (2016) 27:51-103. doi: 10.1007/7854_2015_390

6. Johnson ZV, Young LJ. Oxytocin and vasopressin neural networks: implications for social behavioral diversity and translational neuroscience. Neurosci Biobehav Rev. (2017) 76(Pt A):87-98. doi: 10.1016/j.neubiorev.2017.01.034.

7. Dumais KM, Veenema AH. Vasopressin and oxytocin receptor systems in the brain: sex differences and sex-specific regulation of social behavior. Front Neuroendocrinol. (2016) 40:1-23. doi: 10.1016/j.yfrne.2015.04.003 investigators to guide their work and will spark new discussions about the role of the Avp/Avt systems in behavioral regulation. Given the complexity of these systems, the diversity of species studied, and the numerous behaviors they regulate, it appears that even 60 years after the structural definition of Avp, much work still remains to be done.

\section{AUTHOR CONTRIBUTIONS}

All authors listed have made a substantial, direct and intellectual contribution to the work, and approved it for publication.

8. Albers HE. Species, sex and individual differences in the vasotocin/vasopressin system: relationship to neurochemical signaling in the social behavior neural network. Front Neuroendocrinol. (2015) 36:49-71. doi: 10.1016/j.yfrne.2014.07.001

9. Paul MJ, Terranova JI, Probst CK, Murray EK, Ismail NI, de Vries GJ. Sexually dimorphic role for vasopressin in the development of social play. Front Behav Neurosci. (2014) 8:58. doi: 10.3389/fnbeh.2014.00058

10. Rilling JK, Demarco AC, Hackett PD, Chen X, Gautam P, Stair S, et al. Sex differences in the neural and behavioral response to intranasal oxytocin and vasopressin during human social interaction. Psychoneuroendocrinology (2014) 39:237-48. doi: 10.1016/j.psyneuen.2013.09.022

11. Veenema AH, Bredewold R, De Vries GJ. Sex-specific modulation of juvenile social play by vasopressin. Psychoneuroendocrinology (2013) 38:2554-61. doi: 10.1016/j.psyneuen.2013.06.002

Conflict of Interest Statement: The authors declare that the research was conducted in the absence of any commercial or financial relationships that could be construed as a potential conflict of interest.

Copyright (c) 2018 Caldwell and Petrulis. This is an open-access article distributed under the terms of the Creative Commons Attribution License (CC BY). The use, distribution or reproduction in other forums is permitted, provided the original author(s) and the copyright owner(s) are credited and that the original publication in this journal is cited, in accordance with accepted academic practice. No use, distribution or reproduction is permitted which does not comply with these terms. 